\title{
Breast Care
}

Vol. 8, Issue 4, August 2013

\section{Focus: Pathway-Oriented Concepts} in Adjuvant/Neoadjuvant Therapy

Coordination: Kaufmann, B. (Tel Hashomer); Jackisch, C. (Offenbach)

\section{Editorial}

246 Pathway-Oriented Concepts in Adjuvant and Neoadjuvant Breast Cancer Therapy von Minckwitz, G. (Neu-Isenburg/Frankfurt/Düsseldorf); Fontanella, C. (Neu-Isenburg/Udine)

Review Articles

248 Endocrine Resistance in Breast Cancer: Focus on the Phosphatidylinositol 3-Kinase/ Akt/Mammalian Target of Rapamycin Signaling Pathway Peleg Hasson, S.; Rubinek, T.; Ryvo, L.; Wolf, I. (Tel Aviv)

256 Bidirectional Crosstalk between the Estrogen Receptor and Human Epidermal Growth Factor Receptor 2 Signaling Pathways in Breast Cancer: Molecular Basis and Clinical Implications Giuliano, M.; Trivedi, M.; Schiff, R. (Houston, TX)

264 How Long is Enough - Optimal Timing of Anti-HER2/neu Therapy in the Adjuvant Setting in Early Breast Cancer

Pinto, A.C.; de Azambuja, E.; Piccart-Gebhart, M. (Brussels)

Original Articles

270 Male Breast Cancer: 20-Year Survival Data for Post-Mastectomy Radiotherapy

Eggemann, H.; Ignatov, A. (Magdeburg); Stabenow, R. (Berlin); von Minckwitz, G. (Neu-Isenburg); Röhl, F.W.; Hass, P.; Costa, S.-D. (Magdeburg)

\section{Band 8, Heft 4, August 2013}

\section{Schwerpunkt: Signaltransduktionsweg- basierte Konzepte der adjuvanten und neoadjuvanten Brustkrebstherapie Koordination: Kaufmann, B. (Tel Hashomer); Jackisch, C. (Offenbach) \\ Editorial}

246 Signaltransduktionsweg-basierte Konzepte der adjuvanten und neoadjuvanten Brustkrebstherapie von Minckwitz, G. (Neu-Isenburg/Frankfurt/Düsseldorf); Fontanella, C. (Neu-Isenburg/Udine)

Übersichtsarbeiten

248 Endokrine Resistenz bei Brustkrebs: der Phosphatidylinositol-3-Kinase/Akt/Mammalian Target of Rapamycin-Signalweg im Fokus Peleg Hasson, S.; Rubinek, T.; Ryvo, L.; Wolf, I. (Tel Aviv)

256 Gegenseitige Beeinflussung der Signalwege des Östrogenrezeptors und des humanen epidermalen Wachstumsfaktorrezeptors 2 bei Brustkrebs: molekulare Grundlagen und Bedeutung für die Klinik

Giuliano, M.; Trivedi, M.; Schiff, R. (Houston, TX)

264 Wie lang ist lang genug - optimales Timing der Anti-HER2/neu-Therapie im adjuvanten Setting beim frühen Mammakarzinom Pinto, A.C.; de Azambuja, E.; Piccart-Gebhart, M. (Brussels)

Originalarbeiten

270 Das Mammakarzinom beim Mann: Einfluss der postoperativen Bestrahlung auf das Gesamtüberleben bei 20 -jähriger Verlaufsbeobachtung

Eggemann, H.; Ignatov, A. (Magdeburg); Stabenow, R. (Berlin); von Minckwitz, G. (Neu-Isenburg); Röhl, F.W.; Hass, P.; Costa, S.-D. (Magdeburg) 


\section{Breast Care}

Vol. 8, Issue 4, August 2013

276 Is Routine Audiometric Evaluation Necessary in Gynaecologic Tumour Patients Undergoing Chemotherapy?

Fasunla, A.J. (Marburg/Ibadan); Harbeck, N.; Schmalfeld, B.; Berktold, S.; Böhner, C. (Munich); Hundt, W. (Marburg); Wolf, P. (Munich); Steinbach, S. (Marburg)

282 Cross-National Comparison of Medical Costs Shared by Payers and Patients: A Study of Postmenopausal Women with Early-Stage Breast Cancer Based on Assumption Case Scenarios and Reimbursement Fees

Hamada, S.; Hinotsu, S.; Ishiguro, H.; Toi, M.;

Kawakami, K. (Kyoto)

Case Report

290 Inflammatory Myofibroblastic Tumor of the Breast Coexisting with Breast Cancer: A Case Report Li, J.; Yun, W.; Qin, J.; Zhao, J.; Liu, X.; Wu, J.; Ji, M.; Tang, J. (Nanjing)

Review Article

293 Everolimus in Postmenopausal, Hormone Receptor-Positive Advanced Breast Cancer: Summary and Results of an Austrian Expert Panel Discussion

Gnant, M. (Vienna); Greil, R. (Salzburg); Hubalek, M. (Innsbruck); Steger, G. (Vienna)

\section{Clinical Information}

300 Management of Locoregional Recurrence of Breast Cancer with a Focus on HER2-Positive Recurrences

Summary of a Round Table Discussion Jackisch, C. (Offenbach); Schneeweiss, A. (Heidelberg); Untch, M. (Mannheim); Wenz, F. (Berlin-Buch); Bauerfeind, I. (Landshut); Gerber, B. (Rostock)

Expert Discussion

307 Breast Cancer News from ASCO 2013

Chair: Dall, P. (Lüneburg)

Participants: Liedtke, C. (Lübeck); Rack, B. (Munich);

Schütz F. (Heidelberg); Stickeler, E. (Freiburg i.Br.)

\section{Band 8, Heft 4, August 2013}

276 Besteht die Notwendigkeit, gynäkologische Tumorpatientinnen mit derzeit gängigen Chemotherapie-Schemata routinemäßig audiometrisch zu untersuchen?

Fasunla, A.J. (Marburg/Ibadan); Harbeck, N.; Schmalfeld, B.; Berktold, S.; Böhner, C. (Munich); Hundt, W. (Marburg); Wolf, P. (Munich); Steinbach, S. (Marburg)

282 Länderübergreifender Vergleich der von Kassen und Patienten getragenen medizinischen Kosten: Studie über postmenopausale Frauen mit Brustkrebs im Frühstadium basierend auf theoretischen Fallszenarien und Rückerstattungsbeträgen Hamada, S.; Hinotsu, S.; Ishiguro, H.; Toi, M.; Kawakami, K. (Kyoto)

Kasuistik

290 Ein entzuindlicher myofibroblastischer Tumor in der Brust in Koexistenz mit Brustkrebs: Ein Fallbericht

Li, J.; Yun, W.; Qin, J.; Zhao, J.; Liu, X.; Wu, J.; Ji, M.; Tang, J. (Nanjing)

Übersichtsarbeit

293 Everolimus beim fortgeschrittenen postmenopausalen, hormonrezeptorpositiven Mammakarzinom: Ergebnisse und Zusammenfassung einer Expertendiskussion Gnant, M. (Vienna); Greil, R. (Salzburg); Hubalek, M. (Innsbruck); Steger, G. (Vienna)

Klinische Information

300 Behandlung des lokoregionären Rezidivs unter besonderer Berücksichtigung der HER2-positiven Rezidive

Zusammenfassung einer Round-Table-Diskussion Jackisch, C. (Offenbach); Schneeweiss, A. (Heidelberg); Untch, M. (Mannheim); Wenz, F. (Berlin-Buch); Bauerfeind, I. (Landshut); Gerber, B. (Rostock)

Expertenplenum

307 Neuigkeiten vom ASCO 2013 zur Brustkrebsbehandlung Gesprächsleiter: Dall, P. (Lüneburg) Teilnehmer: Liedtke, C. (Lübeck); Rack, B. (Munich); Schütz F. (Heidelberg); Stickeler, E. (Freiburg i.Br.)

\section{KARGER}

Fax +497614520714 Information@Karger.com www.karger.com
(C) 2013 S. Karger GmbH, Freiburg 


\section{Breast Care}

Vol. 8, Issue 4, August 2013

Journal Club

311 Should We Encourage Our Patients to Drink Alcohol?

312 Lymphedema: Patients' Fear Is Often Unsubstantiated

Information from Breast Centers

314 The 'Essen Model' - a Concept of Integrative Oncology for Breast Cancer Patients

Paul, A.E.; Lange, S.; Voiß, P.; Klose, P.; Kümmel, S.; Dobos, G.J. (Essen)

318 Innovations

320 PharmaNews

322 Meetings and Conferences

324 Contents of Forthcoming Issues

299 Imprint
Band 8, Heft 4, August 2013

Journal Club

311 Sollten wir unsere Patientinnen anhalten, Alkohol zu trinken?

312 Lymphödem: Die Angst der Patientinnen ist oft unbegründet

Informationen aus den Brustzentren

314 Das «Essener Modell» - ein Konzept zur integrativ-onkologischen Therapie von Brustkrebspatientinnen

Paul, A.E.; Lange, S.; Voiß, P.; Klose, P.; Kümmel, S.; Dobos, G.J. (Essen)

318 Innovationen

320 PharmaNews

322 Tagungen und Kongresse

324 Themenvorschau

299 Impressum 